Tohoku J. Exp. Med., 2010, 220, 223-228

\title{
D-4F, an Apolipoprotein A-I Mimetic Peptide, Promotes Cholesterol Efflux from Macrophages via ATP-Binding Cassette Transporter A1
}

\author{
Qiong Xie, ${ }^{1}$ Shui-ping $\mathrm{Zhao}^{1}$ and Feng $\mathrm{Li}^{2}$ \\ ${ }^{1}$ Department of Cardiology, The Second Xiang Ya Hospital of Central South University, Changsha, Hunan, P.R. \\ China \\ ${ }^{2}$ Department of Cardiothoracic Surgery, The Second Xiang Ya Hospital of Central South University, Changsha, \\ Hunan, P.R. China
}

\begin{abstract}
Cholesterol efflux is the key step of reverse cholesterol transport and has become a therapeutic target against atherosclerosis. Human apolipoprotein $A-I(a p o A-I)$ is the main protein in high-density lipoprotein (HDL) and has 243 amino acids. ApoA-I mimetic peptides have no sequence homology to apoA-I but possess the class-A amphipathic helical motif that presents in apoA-I lipid binding domains. D-4F is one of the apoA-I mimetic peptides and exerts diverse atheroprotective functions similar to apoA-I. However, the exact role of $\mathrm{D}-4 \mathrm{~F}$ on lipid metabolism in macrophages is not clear yet. Therefore, we studied the effect of D-4F on cholesterol efflux, cAMP levels and expression of ATP-binding cassette transporter A1 (ABCA1) in RAW264.7 mouse macrophages. Cells were incubated with 1, 10, 50 or $100 \mu \mathrm{g} / \mathrm{ml} \mathrm{D-4F}$ for 24 hours, and the cholesterol efflux was assessed. Here, D-4F significantly increased the cholesterol efflux in concentration- and time-dependent manners. Concomitantly, D-4F increased intracellular cAMP levels and the expression levels of ABCA1 mRNA and protein in a dose-dependent manner, consistent with the increase in the cholesterol efflux from macrophages. 8-Br-cAMP (cAMP activator) increased the D-4Fmediated cholesterol efflux by $39 \%$. Moreover, the increases in cholesterol efflux and ABCA1 expression induced by 8-Br-cAMP could be inhibited by the treatment with $\mathrm{H} 89$, a protein kinase A (PKA) inhibitor. In conclusion, these results suggest that the synthetic peptide $\mathrm{D}-4 \mathrm{~F}$ promotes cholesterol efflux in macrophages through the CAMP-PKA-ABCA1 pathway, which may open new avenues for the treatment of atherosclerosis.
\end{abstract}

Keywords: apolipoprotein A-I mimetic peptide; cholesterol efflux; macrophage; cyclic AMP; ATP-binding cassette transporter A1

Tohoku J. Exp. Med., 2010, 220 (3), 223-228. 두 2010 Tohoku University Medical Press

Atherosclerotic cardiovascular disease remains an important cause of mortality and morbidity throughout most of the world. An early hallmark of atherosclerosis is the accumulation of cholesterol-loaded macrophages (foam cells) in the intima of arteries. Removing the excess cholesterol in macrophages, maintaining intracellular cholesterol homeostasis and preventing the formation of macrophagederived foam cells has become an important therapeutic target for the prevention and reversal of atherosclerosis (Cavelier et al. 2006).

Recently, apolipoprotein-based mimetic peptides have become a hot issue in the field of atherosclerosis. One of the most intriguing approaches under investigation is the use of apolipoprotein A-I (apoA-I) mimetic peptides, which mimic the function of apoA-I without bearing any sequence homology to apoA-I (Navab et al. 2005). D-4F, one of the apoA-I mimetic peptides, contains only 18 amino acids, compared with apoA-I which contains 243 amino acids. It is synthesized from D-amino acids and contains a class A amphipathic helix with a polar and a nonpolar face that allows it to bind lipids similar to apoA-I (Navab et al. 2007). Furthermore, it exerts diverse atheroprotective functions. It can improve the anti-inflammatory properties of high-density lipoprotein (HDL), facilitate cholesterol efflux, prevent atherosclerosis in mice and, when administered with a statin, cause regression in old mice (Navab et al. 2004b, 2005). Addition of D-4F to human plasma in vitro reproduces many of the activities seen in vivo (Navab et al. 2006). Thus, the mimetic peptide is increasingly considered as potential effective treatments for atherosclerosis and

Received November 13, 2009; revision accepted for publication February 4, 2010. doi:10.1620/tjem.220.223

Correspondence: Shui-Ping Zhao, Department of Cardiology, The Second Xiangya Hospital of Central South University, middle

Ren-Min road No. 139, Changsha, Hunan 410011, P.R. China.

e-mail: zhaosp@medmail.com.cn, bestxq80@hotmail.com 
related cardiovascular disorders.

However, there is few study about the effects of D-4F on the lipid metabolism of macrophages which play a key role in atherosclerosis. Moreover, it is still unclear whether the mimetic peptide shares the same pathway with apoA-I to modulate cholesterol efflux (Tang et al. 2006). In the present work, we examined the effects of D-4F on cholesterol efflux from RAW264.7 macrophages and investigated the possible mechanism.

\section{Methods}

Peptide Synthesis

D-4F with the primary sequence Ac-DWFKAFYDKVAEKF KEAF- $\mathrm{NH}_{2}$ was synthesized by Fmoc chemistry, using an automatic peptide synthesizer (PE Biosystems, Foster City, CA, USA) according to the procedure as described (Datta et al. 2000). The purity of synthetic peptides (typically $>98 \%$ ) was established by analytical highperformance liquid chromatography (HPLC) and confirmed by mass spectral analysis.

\section{Cell culture}

RAW264.7, a murine macrophage cell line, was maintained in Dulbecco's modified Eagle's medium (DMEM) supplemented with $10 \%$ fetal bovine serum and antibiotics. Cells were grown at $37^{\circ} \mathrm{C}$ and $5 \% \mathrm{CO}_{2}$ in humidified air.

\section{Determination of cAMP Content in Cells}

Cellular cAMP content was measured by using a commercially available ELISA kit (R\&D Systems Inc., Minneapolis, MN, USA) following the manufacturer's instructions.

\section{Cholesterol efflux assays}

Experiments were performed as described by Marcil et al (Marcil et al. 2003) with minor modifications. Cells were plated at a density of $5 \times 10^{5}$ cells $/ \mathrm{mL}$ in 24-well culture dishes. After 24 hours starvation in DMEM containing $2 \mathrm{mg} / \mathrm{ml}$ bovine serum albumin (BSA). Cells were incubated in medium containing $0.5 \mu \mathrm{Ci} /$ well $\left[{ }^{3} \mathrm{H}\right]$-labeled cholesterol and $100 \mu \mathrm{g} / \mathrm{ml}$ oxidized-low density lipoprotein (oxLDL) for 24 hours. Then the cells were incubated in the absence or presence of the peptide $\mathrm{D}-4 \mathrm{~F}$ with various concentrations. In some experiments, cells were treated with $0.5 \mathrm{mM} 8$-Br-cAMP or $20 \mathrm{uM} \mathrm{H89}$ for 12 hours prior and during the process of the cholesterol efflux. Radioactivity in the supernatants and total cell extracts were measured by scintillation counting. Cellular cholesterol efflux was determined as follow: ${ }^{3} \mathrm{H}$ cpm in medium $/\left({ }^{3} \mathrm{H}\right.$ cpm in medium $+{ }^{3} \mathrm{H}$ cpm in cells $)$. The results were expressed as percentage of total radiolabeled cholesterol. All experiments were performed in quadruplicated.

\section{Quantitation of ABCA1 mRNA by real-time RT-PCR}

Total RNA from cell cultures was isolated with the TRIzol reagent (Invitrogen Corporation, Camarillo city, CA, USA). First strand cDNA was synthesized from $1 \mu \mathrm{g}$ total RNA with M-MLV reverse transcriptase (Promega Corporation, Madison, WI city, USA) using oligo $(\mathrm{dT})_{15}$ primers(Promega Corporation, Madison, WI city, USA). SYBR Green I-based quantitative real-time RT-PCRs were carried out on ABI7300 (Applied Biosystems, Foster City, CA, USA), using SYBR ${ }^{\circledR}$ Premix Ex TaqTM II (Takara Bio Inc., Otsu, Japan), ROX Reference Dye, $0.4 \mu \mathrm{M}$ each forward and reverse primers, and $\mathrm{dH}_{2} \mathrm{O}$ in a $20 \mathrm{ul}$ reaction volume. The primers (Takara Bio Inc., Otsu, Japan) used in this experiment are indicated in Table 1 (F: forward, R: reverse). The samples were then subjected to the following PCR conditions: initial heat-denaturing at $95^{\circ} \mathrm{C}$ for 30 s followed by 40 cycles of $95^{\circ} \mathrm{C}$ for $5 \mathrm{~s}, 60^{\circ} \mathrm{C}$ for $31 \mathrm{~s}$ and the $3 \mathrm{rd}$ stage including $95^{\circ} \mathrm{C}$ for $15 \mathrm{~s}$, $60^{\circ} \mathrm{C}$ for $1 \mathrm{~min}$ and $95^{\circ} \mathrm{C}$ for $15 \mathrm{~s}$. Optical detection to evaluate the SYBR Green fluorescence was conducted during the last extension step. Expression of the target gene relative to the endogenous control gene ( $\beta$-actin), was calculated using the difference in threshold cycle $\left(\Delta \mathrm{C}_{\mathrm{t}}=\mathrm{C}_{\mathrm{t}}\right.$ target $-\mathrm{C}_{\mathrm{t}}$ control $)$ method, in which the relative expression equaled $2^{-\Delta(\Delta \mathrm{Ct})} \Delta \Delta \mathrm{C}_{\mathrm{t}}=\Delta \mathrm{C}_{\mathrm{t}}$ target $-\Delta \mathrm{C}_{\mathrm{t}}$ untreated). Sample data were normalized to $\beta$-actin mRNA and all data are presented as the fold change relative to the control.

\section{Western Blotting for ABCA1 protein}

Cultured cells were lysed in radioimmunoprecipitation assay(RIPA) buffer(10 mmol/L Tris [pH 7.4], $150 \mathrm{mmol} / \mathrm{L} \mathrm{NaCl}, 1 \%$ Nonidet P-40, $1 \%$ sodium deoxycholate, $0.1 \%$ sodium dodecyl sulfate, Complete protease inhibitors from Roche). Total cell lysates were separated by SDS-PAGE and the proteins were transferred on PVDF membranes. The blots were blocked with 5\% non-fat dry milk in Tris-buffered saline (TBS) for 1 hour at room temperature and subsequently incubated overnight at $4^{\circ} \mathrm{C}$ with primary antibodies at the appropriate dilution recommended in the product datasheet. Following three washes of 10-15 minutes each with TBST [TBS, $0.1 \%$ (v:v) Tween 20], the blots were incubated with horseradish peroxidase-conjugated goat anti-rabbit IgG (Cell Signaling Technology Inc., Danvers city, MA, USA) in blocking buffer for 1 hour under room temperature. After three washes with TBST, the blots were developed with chemiluminescence reagent and exposed to X-ray. The expression of ATP-binding cassette transporter A1 (ABCA1) (anti-ABCA1, Abcam, Cambridge, UK) was evaluated and compared with the expression of $\beta$-actin (Cell Signaling Technology Inc., Danvers city, MA, USA).

\section{Statistical Methods}

All results are reported as the mean \pm S.D.. Statistical analysis was performed using SPSS version 13.0 software. Differences between the groups were assessed by One-way ANOVA. A value of

Table1. The specific primers used for Quantitative real-time RT-PCRs.

\begin{tabular}{clcc}
\hline Gene & Accession No. & \multicolumn{1}{c}{ Primer } & Product length (bp) \\
\hline \multirow{2}{*}{$\beta$-actin } & NM_007393 & F 5'-ACTGCTCTGGCTCCTAGCAC-3' & 115 \\
& & R 5'-ACATC TGCTGGAAGGTGGAC-3 & 185 \\
\hline \multirow{2}{*}{ ABCA1 } & NM-013454 & F 5'-AATTCTCAAGTGCAAACACTTCTGG-3' & \\
\hline
\end{tabular}


A

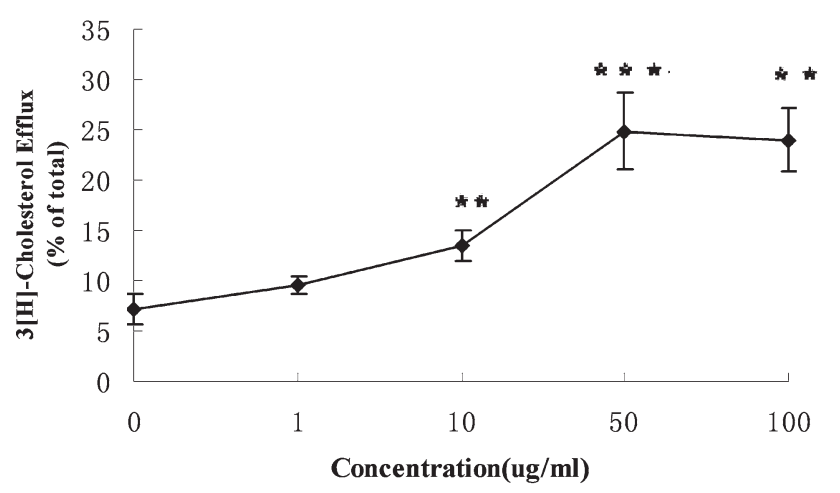

B
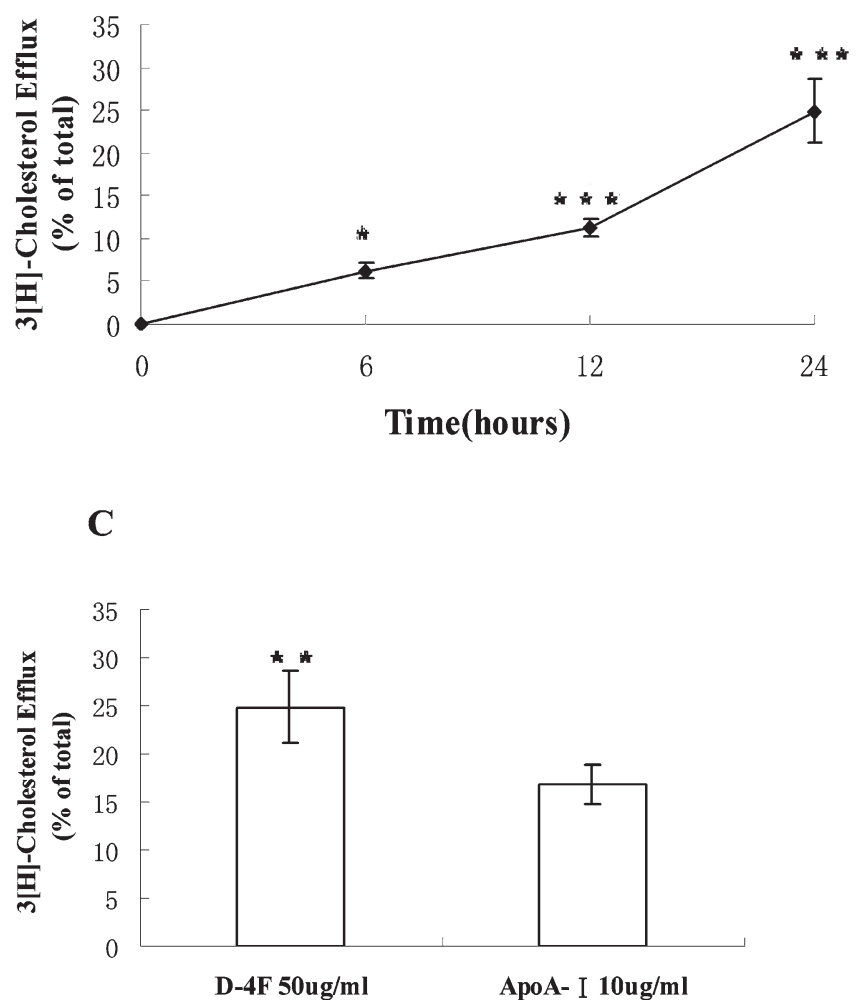

Fig. 1. Effect of the peptide D-4F on cellular cholesterol effl ux in macrophages. (A) D-4F-dose response: $\left.{ }^{3} \mathrm{H}\right]$-cholesterol pre-labeled macrophages were incubated for $24 \mathrm{~h}$ at $37^{\circ} \mathrm{C}$ in DMEM containing different concentrations of D-4F; (B) Time course of cholesterol efflux: macrophages were incubated in DMEM medium containing the peptide $\mathrm{D}-4 \mathrm{~F}(50 \mu \mathrm{g} / \mathrm{ml})$. Samples were taken at different incubation time points. The cholesterol efflux data represents the mean \pm S.D. from quadruplicated wells $(*: P<0.05$ vs control, **: $P<0.01$ vs control, ***: $P<0.001$ vs control); (C) After $24 \mathrm{~h}$ incubation with $50 \mu \mathrm{g} / \mathrm{ml} \mathrm{D}-4 \mathrm{~F}$ or 10 $\mu \mathrm{g} / \mathrm{ml}$ apoA-I, cholesterol efflux was determined as decribed in Method. All data represents the mean \pm S.D. from quadruplicate wells. (**: $P<0.01$ vs $10 \mu \mathrm{g} / \mathrm{ml}$ apoA-I)
$P<0.05$ was considered to be statistically significant.

\section{Results}

$\left[{ }^{3} H\right]$-cholesterol efflux mediated by the peptide $D-4 F$ from macrophages under baseline conditions

In the present study, cholesterol efflux mediated by D-4F from macrophages was measured in cells labeled with $\left[{ }^{3} \mathrm{H}\right]$ cholesterol delivered via oxLDL uptake. Only $7.1 \pm$ $0.45 \%$ of total $\left[{ }^{3} \mathrm{H}\right]$ cholesterol was detectable after 24 hours in the absence of cholesterol acceptors. Compared with the control group, a nearly 2 -fold increase in $10 \mu \mathrm{g} / \mathrm{ml} \mathrm{D}-4 \mathrm{~F}$ mediated cholesterol efflux was seen after 24 hours, where-

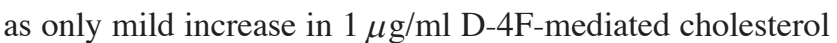
efflux was observed $(9.53 \pm 0.90 \%)$. Meanwhile, as shown in Fig. 1A, this stimulation of cholestrol efflux was dosedependent and reached a plateau at D-4F $50 \mu \mathrm{g} / \mathrm{ml}$. We next investigated the time course of $50 \mu \mathrm{g} / \mathrm{ml} \mathrm{D}-4 \mathrm{~F}$-induced increases of cholesterol efflux. As shown in Fig. 1B, cholesterol efflux to D-4F was first detectable after $6 \mathrm{~h}$ and continued to increase throughout the 24-h efflux period. At different time points, cholestrol efflux rate was $6.25 \pm 1.00$ $\%$ (6 h), $11.24 \pm 1.11 \%(12 \mathrm{~h})$, and $24.86 \pm 3.73 \%$ (24 h), respectively. The cholesterol efflux to D-4F from macrophages was similar to that of apoA-I. However, under the same condition, D-4F $(50 \mu \mathrm{g} / \mathrm{ml})$ is more efficient than apoA-I (10 $\mu \mathrm{g} / \mathrm{ml})$ to induce cholesterol efflux (Fig. 1C).

The effect of the D-4F on intracellular $c A M P$ and $A B C A 1$ expression levels

Experiments were carried out to test whether the enhancement of cholesterol efflux mediated by D-4F correlates with the elevation of the cellular cAMP and ABCA1 levels. Intracellular cAMP levels were measured upon treatment with increased amounts of D-4F $(0,1,10,50$, and $100 \mu \mathrm{g} / \mathrm{ml}$ ). As shown (Fig. 2A), D-4F increased intracellular cAMP levels in a dose-dependent manner, with 50 $\mu \mathrm{g} / \mathrm{ml} \mathrm{D}-4 \mathrm{~F}$ generating a peak of cAMP elevation (2-fold) (Fig. 2B).

We next examined the expression of ABCA1 mRNA in cultured macrophages. Incubating cells with $\mathrm{D}-4 \mathrm{~F}$ of different concentrations $(1,10$, and $50 \mu \mathrm{g} / \mathrm{ml})$ caused enhancement of ABCA1 mRNA expression by 1.7, 1.9 and 2.3 fold, respectively (Fig. 2B). Furthermore, ABCA1 protein levels were highly stimulated by D-4F consistent with the changes in ABCA1 mRNA and cAMP levels (Fig. 2C).

The effect of $c A M P / P K A$ via $A B C A 1$ on $D-4 F$-mediated cholesterol efflux

In order to test the hypothesis that the peptide D-4Fmediated cholesterol efflux involves a cAMP pathway, the macrophages were incubated in the absence or presence of $0.5 \mathrm{mM} 8$-Br-cAMP, a potent cAMP activator, together with $50 \mu \mathrm{g} / \mathrm{ml} \mathrm{D}-4 \mathrm{~F}$. With the treatment of 8-Br-cAMP $(0.5$ $\mathrm{mM}$ ), D-4F-mediated cellular efflux was increased by $39 \%$ as compared with that of the control group (Fig. 3A).

To assess whether the effect of $\mathrm{D}-4 \mathrm{~F}$ is mediated 

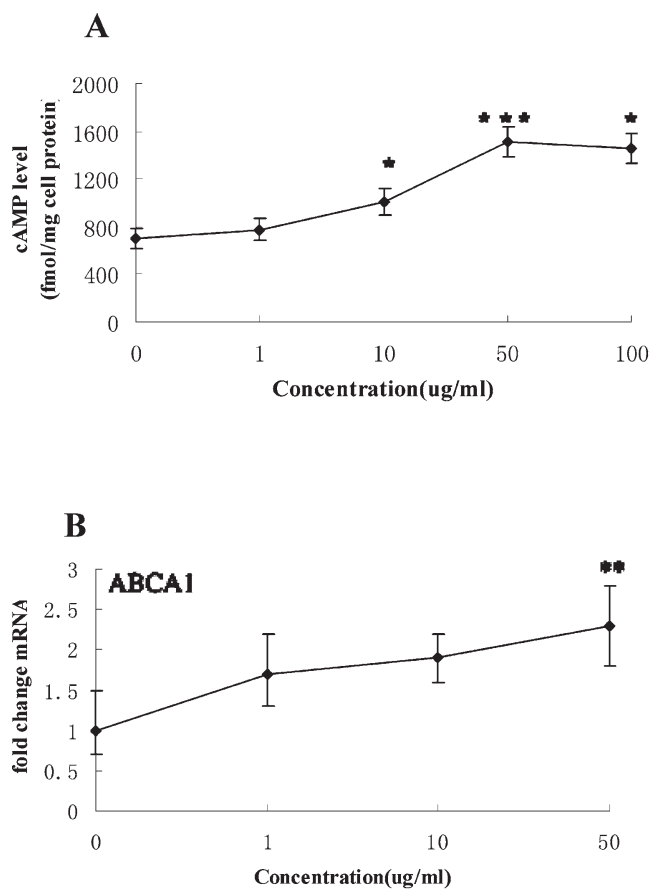

C

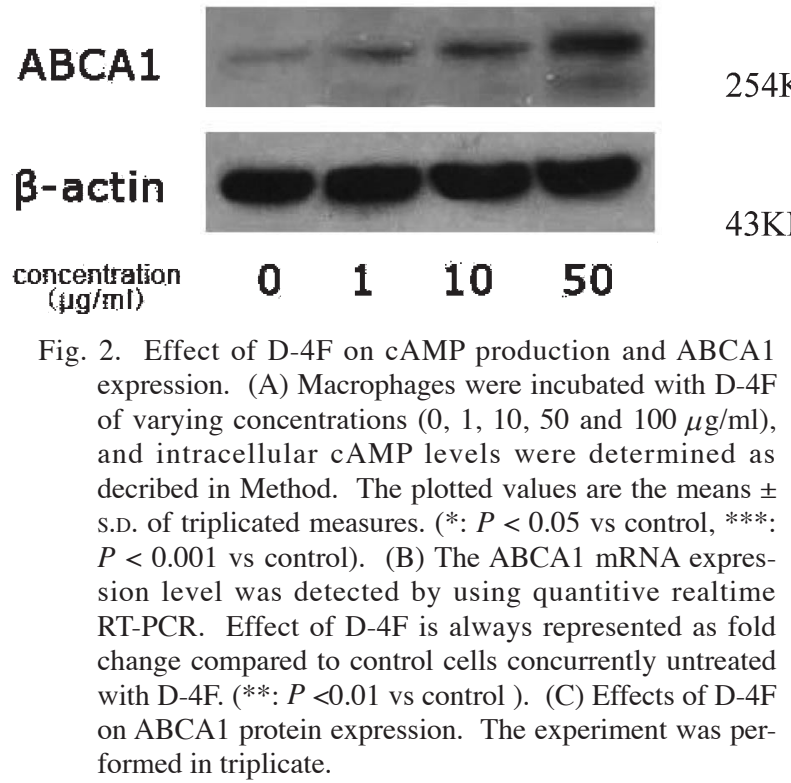

through cAMP-dependent PKA, H89, a specific PKA inhibitor was used. It was observed that H89 was only mildly effective at inhibiting basal cholesterol efflux in the absence of 8 -Br-cAMP $(21.95 \pm 3.00 \%)$. However, when used in combination with 8-Br-cAMP, H89 had the ability to inhibit the 8-Br-cAMP-stimulated efflux by about $37.8 \%$.

We also tested whether the increase in D-4F-mediated cholesterol efflux induced by 8-Br-cAMP was associated with changes in cellular cAMP levels. Macrophages were incubated for 24 hours with 8-Br-cAMP $(0.5 \mathrm{mM})$. The addition of 8-Br-cAMP to oxLDL-treated macrophages resulted in a 2 -fold increase in cellular cAMP levels, while
H89 almost had no effect (Fig. 3B).

In order to establish the relationship of cAMP/PKAdependent pathway with ABCA1 activity, we detected ABCA1 expression by Western blotting analysis and quantitative real-time RT-PCR assays. We observed that 8Br-cAMP dramatically increased ABCA1 mRNA by $520 \%$ (Fig. 3C) and protein expression level (Fig. 3D). These results demonstrated that 12-hour pretreatment with H89 separately did not have an effect on ABCA1 expression and H89 actually inhibited the enhancement of ABCA1 expression due to 8-Br-cAMP.

\section{Discussion}

Reverse cholesterol transport(RCT) is a multistep process by which free cholesterol is transported from the peripheral tissues to the liver, then is removed from the body (Rader et al. 2009). The cholesterol efflux from cells (especially from macrophages) is the first step of RCT and responsible for maintaining cellular cholesterol homeostasis (Yokoyama 2000). As the key step of RCT, cholesterol efflux has become a therapeutic target against atherosclerosis.

One major pathway facilitating the cellular cholesterol and phospholipid efflux in macrophages depends on the presence of lipophilic acceptor particles. It is generally accepted that HDL and apoA-I are the most commom acceptors and can significantly mediate the cholesterol efflux (Ohashi et al. 2005). Recently, attention has been focused on apo A-I mimetic peptides which contain the class A amphipathic helical motif that is responsible for solubilizing phospholipids and cholesterol. It's reported that oral D-4F in apolipoprotein $\mathrm{E}$ (apoE) null mice improved the ability of HDL to promote cholesterol efflux and RCT in macrophages (Navab et al. 2004a). In our experiment D-4F was designed to serve as cholesterol efflux acceptor. We observed the peptide dramatically increased the cholesterol efflux from macrophages in a concentration- and timedependent manner. Thus, we can conclude that small synthetic peptide D-4F containing class A amphipathic helices can mimic apoA-I's ability to promote ABCA1-dependent cholesterol efflux from macrophages.

The importance of ABCA1 pathways in cholesterol efflux and total body lipid homeostasis has been highlighted by the identification of Tangier disease which characterized by marked cholesterol accumulation in macrophages and other reticuloendothelial cells (Cavelier et al. 2006). ABCA1 can be highly regulated at the transcriptional and post- translational levels. The promoter fragment -200 to -80 bp of ABCA1 contains binding motifs for transcription factors which modulates ABCA1 gene expression, notably in response to cellular cAMP(Santamarina-Fojo et al. 2000). cAMP stimulates not only the expression of ABCA1 in marophages but also the phosphorylation of $\mathrm{ABCA} 1$, which involves protein kinase $\mathrm{A}(\mathrm{PKA})($ Haidar et al. 2004). Interestingly, Oram et al observed that cAMP induces apoA-I binding activity and promotes cellular cholesterol 
A

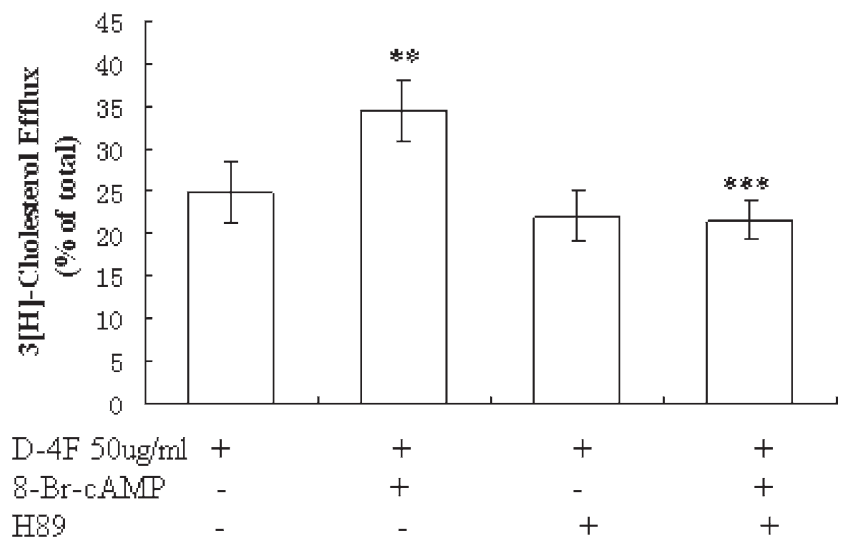

B

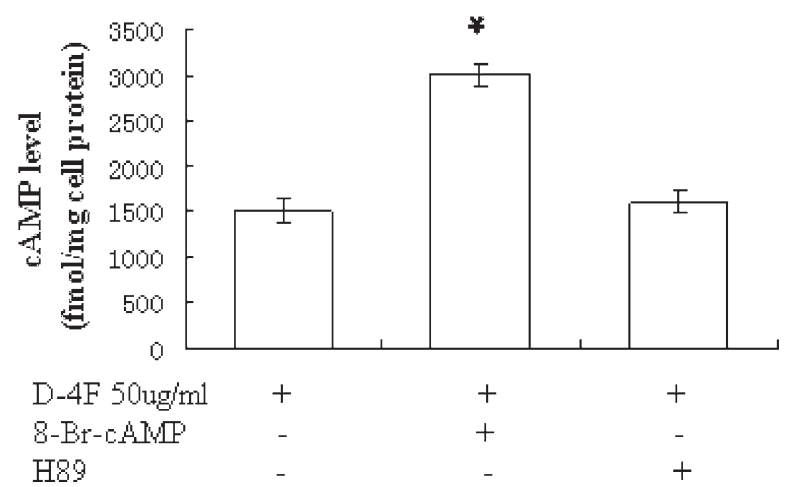

$\mathrm{C}$

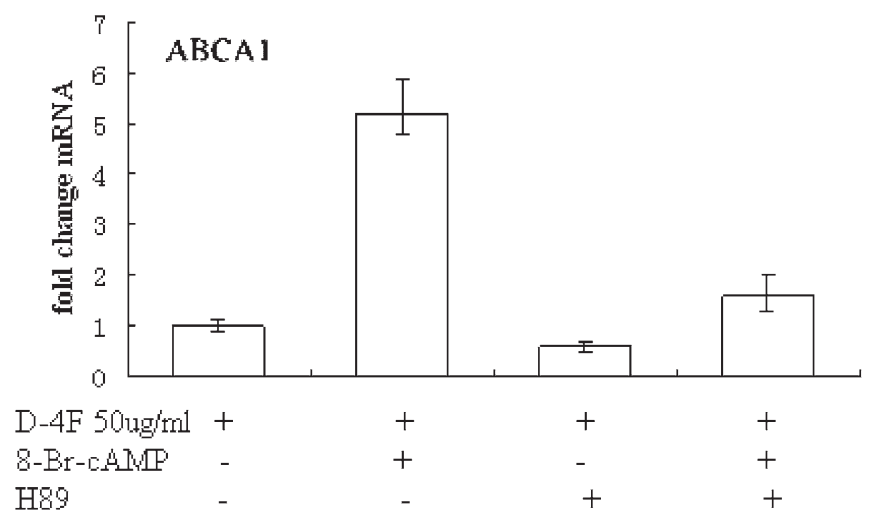

$\mathbf{D}$

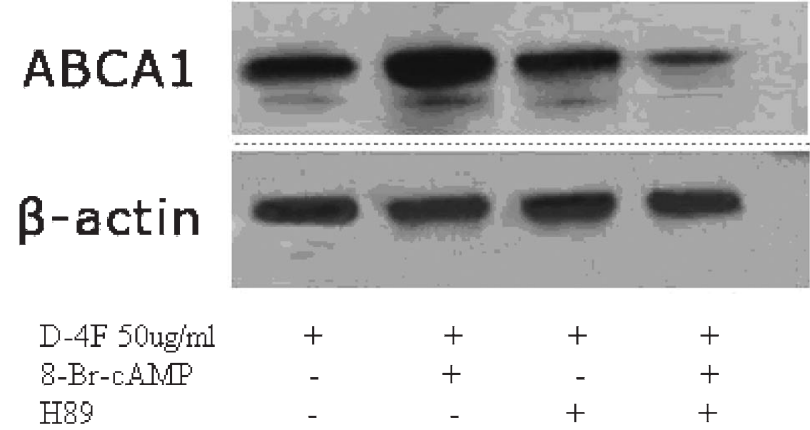

Fig. 3. Effect of 8-Br-cAMP and H89 on D-4F-mediated cellular cholesterol efflux, cellular cAMP level and ABCA1 expression. Cells were treated with 8-Br-cAMP $(0.5 \mathrm{mM})$ or/and $\mathrm{H} 89(20 \mu \mathrm{M}) 12 \mathrm{~h}$ prior to and during efflux. Cells were then incubated with D-4F $(50 \mu \mathrm{g} / \mathrm{ml})$. (A) The radioactivity in the medium and in the cells was determined. Cholesterol effl ux represented the mean \pm S.D. from quadruplicated wells. $* *: P<0.01 \mathrm{vs}$ control, $* * *: P<0.001 \mathrm{vs} \mathrm{8-Br-cAMP} \mathrm{group.}$ (B) The intracellular cAMP levels were determined. Plotted values are the means \pm S.D. of triplicated values. $*: P<0.05$ vs control. (C) ABCA1 mRNA expression was investigated by realtime RT-PCR as described in Methods. (D) Protein levels of ABCA1 in macrophages were detected by western blot analysis. The experiment was performed in triplicate.

efflux via ABCA1 protein in macrophages(Oram et al. 2000). In addition, B.Haidar et al demonstrated that the cAMP/ PKA-dependent pathway plays a pivotal role in ABCA1 phosphorylation and modulates cellular lipid efflux mediated by apoA-I in fibroblasts (Haidar et al. 2002). These studies all have documented that apoA-I activates cAMP signalling and thereby the phosphorylation of ABCA1.

In the present study, we demonstrated that $\mathrm{D}-4 \mathrm{~F}$ modulated the expression of ABCA1 mRNA and protein levels in a dose-dependent manner, consistent with the changes of cholesterol efflux in macrophages. Meanwhile, we observed that D-4F increased intracellular cAMP levels, consistent with the increases in the level of ABCA1 mRNA and protein expression and consequently the cellular cholesterol efflux. Therefore, we assume that $\mathrm{D}-4 \mathrm{~F}$ shares the similar signaling pathway with apoA-I to modulate cholesterol efflux and ABCA1 expression in macrophages. Then we examined the effects of 8-Br-cAMP and H89 on cholesterol efflux and ABCA1 expression mediated by $50 \mu \mathrm{g} / \mathrm{ml}$ D-4F. Our result showed that 8-Br-cAMP was a strong modulator of cholesterol efflux and ABCA1 expression in mouse macrophages. Moreover, H89 could remarkably inhibit the 8-Br-cAMP-inducible component of cholesterol efflux and ABCA1 expression levels, although it mildly affected basal cholesterol efflux and ABCA1 expression in the absence of 8-Br-cAMP. These results indicate that the effect of 8-Br-cAMP on cellular cholesterol efflux is secondary to enhanced ABCA1 expression and phosphorylation by PKA. Therefore, it seems reasonable to suppose that there exist a cAMP-PKA-ABCA1 pathway in cholesterol efflux mediated by D-4F. However, Tang et al. (2004, 2006) have documented that exposing ABCA1-expressing cells to apoA-I or its mimetic peptides activates the Janus Activating Kinase 2 (JAK2), while inhibiting or ablating JAK2 dramatically reduces binding of amphipathic helices 
to ABCA1. These observations lead us to propose that the amphipathic helices coordinate the activity of ABCA1 by several distinct mechanisms that are likely to involve different cell surface binding sites.

In summary, we have found that D-4F not only serves as a simple cholesterol acceptor, but also plays an important role in cholesterol efflux from macrophages by inducing the intracellular cAMP release and then modulating ABCA1 expression via a PKA-dependent manner. This effect requires initial $\mathrm{D}-4 \mathrm{~F}$ binding to the ABCA1 transporter, which leads to activation of cAMP production, and subsequent PKA-mediated ABCA1 phosphorylation. Further studies of exact molecular mechanism of D-4F in mediating cellular signaling and its direct interaction with ABCA1 in macrophages are needed. These highlighted results support the notion that the synthetic peptide D-4F has therapeutic potential in reducing common pathological features of atherosclerosis.

\section{Acknowledgments}

This work was supported by the National Natural Science Foundation of China(Grant No. 30770857) and Key Clinical Program of the Ministry of Health. The authors would like to thank Professor Jian-fang Chen for cholesterol assays.

\section{References}

Cavelier, C., Lorenzi, I., Rohrer, L. \& von Eckardstein, A. (2006) Lipid efflux by the ATP-binding cassette transporters ABCA1 and ABCG1. Biochim. Biophys. Acta, 1761, 655-666.

Datta, G., Chaddha, M., Garber, D.W., Chung, B.H., Tytler, E.M., Dashti, N., Bradley, W.A., Gianturco, S.H. \& Anantharamaiah, G.M. (2000) The receptor binding domain of apolipoprotein E, linked to a model class A amphipathic helix, enhances internalization and degradation of LDL in fibroblasts. Biochemistry, 39, 213-220.

Haidar, B., Denis, M., Krimbou, L., Marcil, M. \& Genest, Jr. J. (2002) cAMP induces ABCA1 phosphorylation activity and promotes cholesterol efflux from fibroblasts. J. Lipid Res., 43, 2087-2094.

Haidar, B., Denis, M., Marcil, M., Krimbou, L. \& Genest, Jr. J. (2004) Apolipoprotein A-I activates cellular cAMP signaling through the ABCA1 transporter. J. Biol. Chem., 279, 99639969.

Marcil, M., Bissonnette, R., Vincent, J., Krimbou, L. \& Genest, J. (2003) Cellular phospholipid and cholesterol efflux in highdensity lipoprotein deficiency. Circulation, 107, 1366-1371.

Navab, M., Anantharamaiah, G.M., Reddy, S.T., Hama, S., Hough, G., Grijalva, V.R., Wagner, A.C., Frank, J.S., Datta, G.,
Garber, D. \& Fogelman, A.M. (2004a) Oral D-4F causes formation of pre-beta high-density lipoprotein and improves highdensity lipoprotein-mediated cholesterol efflux and reverse cholesterol transport from macrophages in apolipoprotein E-null mice. Circulation, 109, 3215-3220.

Navab, M., Anantharamaiah, G.M., Reddy, S.T., Hama, S., Hough, G., Grijalva, V.R., Yu, N., Ansell, B.J., Datta, G., Garber, D.W. \& Fogelman A.M. (2005) Apolipoprotein A-I mimetic peptides. Arterioscler. Thromb. Vasc. Biol., 25, 1325-1331.

Navab, M., Anantharamaiah, G.M., Reddy, S.T., Van Lenten, B.J., Ansell, B.J., Fonarow, G.C., Vahabzadeh, K., Hama, S., Hough, G., Kamranpour, N., Berliner, J.A., Lusis, A.J. \& Fogelman, A.M. (2004b) The oxidation hypothesis of atherogenesis: the role of oxidized phospholipids and HDL. J. Lipid Res., 45, 993-1007.

Navab, M., Anantharamaiah, G.M., Reddy, S.T., Van Lenten, B.J., Buga, G.M. \& Fogelman, A.M. (2007) Peptide Mimetics of Apolipoproteins Improve HDL Function. J. Clin. Lipidol., 1, 142-147.

Navab, M., Anantharamaiah, G.M., Reddy, S.T., Van Lenten, B.J., Datta, G., Garber, D. \& Fogelman, A.M. (2006) Potential clinical utility of high-density lipoprotein-mimetic peptides. Curr. Opin. Lipidol., 17, 440-444.

Ohashi, R., Mu, H., Wang, X., Yao, Q. \& Chen, C. (2005) Reverse cholesterol transport and cholesterol efflux in atherosclerosis. QJM, 98, 845-856.

Oram, J.F., Lawn, R.M., Garvin, M.R. \& Wade, D.P. (2000) ABCA1 is the cAMP-inducible apolipoprotein receptor that mediates cholesterol secretion from macrophages. J. Biol. Chem., 275, 34508-34511.

Rader, D.J., Alexander, E.T., Weibel, G.L., Billheimer, J. \& Rothblat, G.H. (2009) The role of reverse cholesterol transport in animals and humans and relationship to atherosclerosis. J. Lipid Res., 50 Suppl, S189-S194.

Santamarina-Fojo, S., Peterson, K., Knapper, C., Qiu, Y., Freeman, L., Cheng, J.F., Osorio, J., Remaley, A., Yang, X.P., Haudenschild, C., Prades, C., Chimini, G., Blackmon, E., Francois, T., Duverger, N., Rubin, E.M., Rosier, M., Denefle, P., Fredrickson, D.S. \& Brewer, H.B.Jr. (2000) Completegenomic sequence of the human ABCA1 gene: analysis of the human and mouse ATP-binding cassette A promoter. Proc. Natl. Acad. Sci. USA, 97, 7987-7992.

Tang, C., Vaughan, A.M., Anantharamaiah, G.M. \& Oram, J.F. (2006) Janus kinase 2 modulates the lipid-removing but not protein-stabilizing interactions of amphipathic helices with ABCA1. J. Lipid Res., 47, 107-114.

Tang, C., Vaughan, A.M. \& Oram, J.F. (2004) Janus kinase 2 modulates the apolipoprotein interactions with $\mathrm{ABCA} 1$ required for removing cellular cholesterol. J. Biol. Chem., 279, 76227628.

Yokoyama, S. (2000) Release of cellular cholesterol: molecular mechanism for cholesterol homeostasis in cells and in the body. Biochim. Biophys. Acta, 1529, 231-244. 